\title{
Pentaquark Search at CLAS
}

\author{
Patrizia Rossi \\ on behalf of the CLAS Collaboration \\ Laboratori Nazionali di Frascati - INFN \\ Via Enrico Fermi 40 - 00044 Frascati (Italy) \\ e-mail:patrizia.rossi@lnf.infn.it
}

In 2004 a second-generation experimental program has been undertaken at Jefferson Laboratory (JLab), to try to find a definite answer to whether the $\Theta^{+}$and other 5-quark baryons exist. Here the results of CLAS high-statistics experiments are reported.

\section{Introduction}

Quantum Chromodynamics is the underlying theory of the strong interaction but its realization in hadronic physics is not completely understood. In fact, there is no explanation why the observed hadrons combine only in two colorless configurations: mesons $(q \bar{q})$ and baryons $(q q q)$. Exotics, i.e. particles with more complex quark configurations, have been proposed since the early 70's. In 1997 an antidecuplet of 5-quarks baryons with as lowest mass member an isosinglet state, the $\Theta^{+}$, with quark configuration $(u u d d \bar{s})$ giving $\mathrm{S}=+1$, mass $\sim 1.54 \mathrm{GeV}$ and width $\sim 15 \mathrm{MeV}$ was predicted [2].

Experimental evidence for the $\Theta^{+}$, was reported for the first time by the LEPS Collaboration [3]. Evidence for an additional narrow exotic $S=-2$ state, called the $\Xi^{--}$with mass $\sim 1862 \mathrm{MeV}$ was reported by the NA49 Collaboration [4]. Immediately after the LEPS announcement, several other groups analyzed old data, and found the exotic baryon $\Theta^{+}$in both $p K^{0}$ and $n K^{+}$decay channels $[5,6,7,8,9,10,11,12,13]$. There were questions about some of these observations but given the weight of positive supporting evidence reported, the Particle Data Group assigned 3-star status to the $\Theta^{+}$in its 2004 edition. Nevertheless, the relatively small statistical significance of every measurement, the discrepancy in mass determination, and above all, the null results reported by a similar number of high statistics and high energy experiments [14] have driven a second-generation experimental program in different laboratories, among those JLab.

\section{The CLAS program at JLab}

During the year 2004-2005, new dedicated experiments have been performed in Hall B at Jefferson Laboratory (JLab) using the CLAS detector [15] with the aim of improving the statistical accuracy, by at least one order of magnitude, of the two positive published results $[6,7]$ on the $\Theta^{+}$pentaquark obtained from existing CLAS data, and to look for the $\Xi^{--}$state. Detector calibration and data quality checks have been carefully undertaken in order to achieve resolutions on the order of a few $\mathrm{MeV}$ for calculated invariant masses and on the order of $\sim 10 \mathrm{MeV}$ for missing masses.

\section{G10 experiment: search for pertaquarks using a deuteron target}

This experiment ran using a $24 \mathrm{~cm}$ long liquid deuterium target and tagged photons in the energy range $(0.8-3.59) \mathrm{GeV}$. An integrated luminosity of $50 p b^{-1}$ was achieved. 


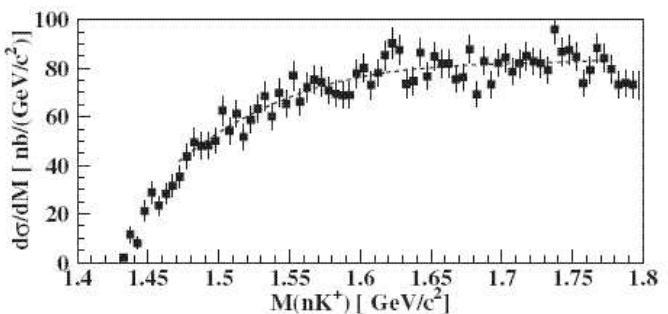

Figure 1: The cross section per mass bin of the $\gamma d \rightarrow p K^{-} \Theta^{+}$reaction.

This reaction channel, with the $\Theta^{+}$decaying into $K^{+} n$, requires the detection of one proton, one $K^{+}$, and one $K^{-}$. The missing mass of the $\gamma d \rightarrow p K^{-} K^{+} X$ reaction was required to be within $\pm 3 \sigma$ of the neutron mass and the missing momentum was required to be greater than $0.20 \mathrm{GeV} / \mathrm{c}$ in order to remove spectator neutrons. Events corresponding to $\phi$-meson production were cut by requiring the $K^{+} K^{-}$mass to be above $1.06 \mathrm{GeV} / c^{2}$, and, events corresponding to the $\Lambda(1520)$ were cut by requiring $1.495 \mathrm{GeV} / c^{2}<M\left(p K^{-}\right)<1.545 \mathrm{GeV} / c^{2}$. The resulting invariant-mass spectrum of the $n K^{+}$system, corrected for the CLAS detector acceptance and normalized by the luminosity, is shown in Fig. 1. These data show no evidence for a narrow peak near $1.54 \mathrm{GeV} / c^{2}$.

\subsubsection{The $\gamma d \rightarrow \Theta^{+} \Lambda$ reaction channel}

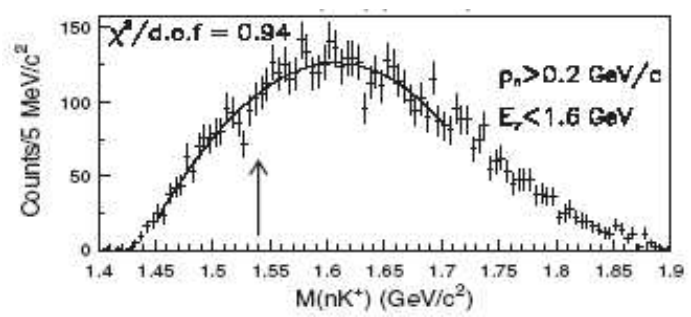

Figure 2: Invariant mass distribution of the $n K^{+}$system for the $\gamma d \rightarrow \Lambda K^{+} n$ reaction after the $E_{\gamma}<1.6 \mathrm{GeV}$ and $p_{n}>0.2 \mathrm{GeV} / \mathrm{c}$ kinematical cuts were applied. The thirdorder polynomial fit used for the upper limit estimate is shown.

The $\gamma d \rightarrow \Theta^{+} \Lambda$ reaction channel has very interesting features: $i$ ) the strangeness content of the final state is well-defined thanks to the presence of the $\Lambda$ particle which has strangeness $S=-1$; ii) the presence of only one $K^{+}$and no $K^{-}$in the final state allows us to identify it without the need of cutting on competing channels $(\phi, \Lambda(1520))$, and iii) kinematical reflections in the NK invariant mass spectrum are excluded.

To analyze this channel, with the $\Theta^{+}$decaying into $\left(K^{+} n\right), p, K, \pi$ were identified using momentum and time-of-flight information. After removing the background under the kaon mass peak due to $p$ or $\pi$ uncorrectly identified as $K$, exclusive $K^{+} n \pi^{-} p$ events were selected by computing the missing mass of the $K^{+} p \pi^{-} X$ system and choosing the events lying within $\pm 3 \sigma$ around the neutron peak. The contribution of the $\Sigma^{-}$decaying weakly into $n \pi^{-}$is removed after applying the $\Lambda$ selection cut on the $p \pi^{-}$invariant mass. After selecting the $\Lambda n K^{+}$events, the $\Theta^{+}$signal was searched for in the invariant mass of the $n K^{+}$system. Since the $n K^{+}$mass spectrum did not show any evident structure, further kinematical cuts have been applied, based upon the indications in [16], in order to try to increase a possible $\Theta^{+}$signal over the non-resonant $n K^{+}$background: i) non-spectatorneutron cut, and ii) photon-energy cut. Under these stringent kinematic conditions still no narrow peaks with statistical relevance were observed in the mass region around 1.54 $\mathrm{GeV} / \mathrm{c}^{2}$. The spectrum is shown in Fig. 2, where the kinematic requirements $p_{n}>0.2$ $\mathrm{GeV} / \mathrm{c}$ and $E_{\gamma}<1.6 \mathrm{GeV}$ are applied. 


\subsection{G11 experiment: search for pentaquarks using a proton target}

In this experiment data were taken using a $40 \mathrm{~cm}$ length liquid hydrogen target and photons in the energy range $(1.6-3.8) \mathrm{GeV}$. An integrated luminosity of $\sim 70 \mathrm{pb}^{-1}$ was achieved.

\subsubsection{The $\gamma p \rightarrow \bar{K}^{0} \Theta^{+}$reaction channel}

The exclusive reactions $\gamma p \rightarrow \bar{K}^{0} K^{+} n$ and $\gamma p \rightarrow \bar{K}^{0} K^{0} p$ were studied to look for evidence of the reaction $\gamma p \rightarrow \bar{K}^{0} \Theta^{+}$. The reactions have been isolated by detecting the $K^{+}$and proton directly, the neutral kaon via its decay $K_{s} \rightarrow \pi^{+} \pi^{-}$, and the neutron or neutral kaon via the missing mass technique. Reactions involving hyperon decays which contribute to the same final states were removed in the analysis. The resulting $n K^{+}$and $p K^{0}$ invariant mass distributions was found smooth and structureless. In particular, no evidence for a peak or an enhancement was observed at masses near $1540 \mathrm{MeV}$. For the reaction channel $\gamma p \rightarrow \bar{K}^{0} K^{+} n$ a comparison with the published results by the SAPHIR Collaboration [8] has been done using, in the analysis, the same cuts reported in Ref. [8]. The resulting mass distribution is shown in the inset of Fig. 3. It remains smooth and structureless.

\subsubsection{The $\gamma p \rightarrow \Theta^{++} p$ reaction channel}

The putative reaction $\gamma p \rightarrow K^{-\Theta^{++}} \rightarrow$ $K^{-} p K^{+}$was studied by the CLAS Collaboration in two ways: in the first case, three final state hadrons, $p, K^{-}$and $K^{+}$were detected while in the second case only a $p K^{+}$ pair were required and the $K^{-}$was identified by missing mass reconstruction. After that, cuts were applied in the $p K^{-}$and $K^{+} K^{-}$mass spectra to eliminate the contribution of the $\Lambda(1520)$ and $\phi(1020)$, respectively. The $p K^{+}$mass spectra after all cuts were applied didn't shown any evidence for any narrow structures that could be interpreted as a $\Theta^{++}$resonance

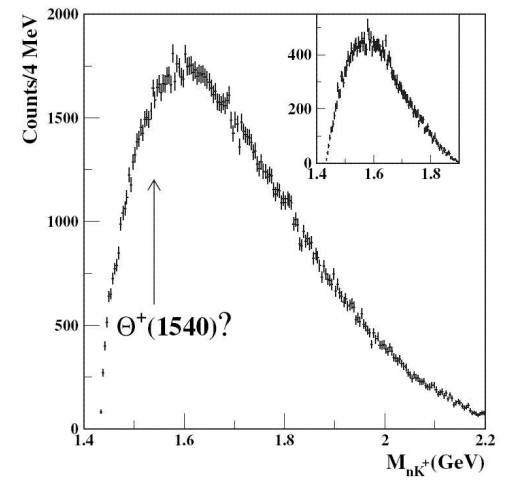

Figure 3: The $n K^{+}$invariant mass distribution for $\gamma p \rightarrow \bar{K}^{0} K^{+} n$ after all cuts. The inset shows the $n K^{+}$mass distribution with specific cuts to reproduce the SAPHIR analysis [8].

\section{Cross section upper limit}

Since no signal was found in any of the measured reactions, an upper limit for the $\Theta^{+}$ and $\Theta^{++}$production cross section was extracted. The $N K$ invariant mass spectrum, acceptance corrected, was fit with the sum of a narrow Gaussian function and a polynomial that parameterize, respectively, the $\Theta^{+}\left(\Theta^{++}\right)$contribution and a smooth background. The signal and background yields were then used to evaluate an upper limit at $95 \%$ confidence level using the Feldman-Cousins method [17]. For details of the followed procedure see Ref. $[18,19,20,21]$. The final results are reported in Table 1.

\section{Outlook and conclusions}


In the year 2004 a comprehensive program to search for pentaquarks in high statistics and high resolution experiments has been started by the CLAS Collaboration at Jefferson Lab. No evidence for pentaquark signals has been found in the photoproduction channels studied so far. From these results, CLAS sets upper limits on photoproduction cross sections from proton and neutron tar-

\begin{tabular}{|l|r|}
\hline Reaction & $\sigma_{U L}$ (95\% C.L.) \\
\hline$\gamma n(p) \rightarrow p K^{-} \Theta^{+}$ & $<3 \mathrm{nb}$ \\
\hline$\gamma d \rightarrow \Theta^{+} \Lambda$ & $<5 \mathrm{nb}$ \\
\hline$\gamma p \rightarrow K^{0} \Theta^{+}$ & $<1 \mathrm{nb}$ \\
\hline$\gamma p \rightarrow \Theta^{++} p$ & $<0.15 \mathrm{nb}$ \\
\hline
\end{tabular}

Table 1: Cross section upper limit at 95\% C.L. for the measured reactions.

gets on the order of a few nb or less. The outlook for the $\Theta^{+}$looks bleak, anyhow it seems that we have to wait a little bit more to put the last word on the pentaquark existence. In fact: $i$ ) the data are still contradictory (LEPS Collaboration has been able to reproduce a peak in a "repeat" measurement); ii) analysis, using higher statistics data, from some experiments that initially claimed the pentaquark, are still in progress (ZEUS, COSY); iii) some theories are able to explain the negative results obtained by CLAS [22, 23, 24]. In the meanwhile, the Particle Data Group assigned 1-star status to the $\Theta^{+}$in its 2006 edition.

\section{References}

[1] Slides: http://indico.cern. ch/contributionDisplay.py? contribId=181\&sessionId=6\&conf Id=9499

[2] D. Diakonv, V. Petrov, M.V. Polyakov, Z. Phys. A359 305 (1997).

[3] T. Nakano et al., Phys. Rev. Lett. 91012002 (2003).

[4] C. Alt et al., Phys. Rev. Lett. 92042003 (2004).

[5] V. Barmin et al., Phys. Atom. Nucl. 661715 (2003).

[6] S. Stepanyan et al., Phys. Rev. Lett. 91252001 (2003).

[7] V. Koubarovsky et al., Phys. Rev. Lett. 92032001 (2004).

[8] J. Barth et al., Phys. Lett. B572 127 (2003).

[9] A. Airapetian et al., Phys. Lett. B585 213 (2004).

[10] A.E. Asratyan, A.G. Dolgolenko, M.A. Kubantsev, Phys. Atom. Nucl. 67682 (2004).

[11] A. Aleev et al., Phys.Atom.Nucl. 68974 (2005); Yad.Fiz. 681012 (2005).

[12] M. Abdel-Bary et al., Phys. Lett. B595 127 (2004).

[13] S. Chekanov et al., Phys. Lett. B591 7 (2004).

[14] J.Z. Bai et al., Phys.Rev. D70 012004 (2004). K.T. Knoepfle et al., J.Phys. G30 S1363 (2004); M.I. Adamovich et al., Phys.Rev. C70 022201 (2004); C. Pinkeburg et al., Eur. Phys. J. C37 91 (2004); BABAR, arXiv:hep-ex/0408064 (2004); Yu.M. Antipov et al., Eur. Phys. J. A21 455 (2004); S. Schaet et al., Phys. Lett. B599 (2004); CDF, E690, LEP, Focus, HyperCP (QNP2004 conference presentation).

[15] B. Mecking et al., Nucl. Inst. \& Meth. A503/3 513 (2003).

[16] V. Guzey, Phys. Rev. C69 173 (2004).

[17] G.D. Feldman and R.D. Cousins, Phys. Rev. D57 3873 (2003).

[18] B. McKinnon et al., Phys. Rev. Lett. 96212001 (2006).

[19] S. Niccolai et al., Phys. Rev. Lett. 97032001 (2006).

[20] M. Battaglieri et al., Phys. Rev. Lett. 96042001 (2006) ; R. De Vita et al., Phys. Rev. D74 032001 (2006).

[21] V. Kubarovsky et al., Phys. Rev. Lett. 97102001 (2006).

[22] S. I. Nam, A. Hosaka, H.-C. Kim, Phys. Lett. B633 483 (2006).

[23] V. Guzey, arXiv:hep-ph/0608129 (2006).

[24] M. Amarian, D. Diakonov, M. Poliakov, arXiv:hep-ex/0612150 (2006). 